\title{
SHORT TEXT TOPIC MODELING WITH EMPIRICAL LEARNING
}

\author{
Supriya A. Kinariwala \\ Department of Computer Science and Engineering \\ Marathwada Institute of Technology, Aurangabad, Maharashtra, INDIA \\ sakinariwala@gmail.com \\ Sachin N. Deshmukh \\ Department of Computer Science and IT \\ Dr. Babasaheb Ambedkar Marathwada University, Aurangabad, Maharashtra, INDIA \\ sndeshmukh@hotmail.com
}

\begin{abstract}
In the present modern digital era, use of social media has been increasing exponentially. People have started using short text for expressing their thoughts. Social media websites like Twitter, Facebook are generating vast amount of short text at every second that reveals good knowledge of real time information. Extensive research is going on to discover knowledge from it. Short text is very sparse and ambiguous; hence there is a big challenge to find latent topics from it. This can be resolved by using unsupervised machine learning approach referred as topic modeling. This paper covers various topic modeling methods like Latent Dirichlet Allocation (LDA), Non-negative Matrix Factorization (NMF), and Semantics-assisted Non-negative Matrix Factorization (SeaNMF) and their comparative analysis. These three methods have been tested on $\mathrm{ABCNews}$ headline dataset, results have been analyzed using average Normalized Google Distance (NGD) score; which is $67.88 \%, 58.60 \%, 59.32 \%$ for SeaNMF, NMF and LDA respectively. The quantitative result shows that more meaningful and semantically similar words are clustered under each topic by SeaNMF model.
\end{abstract}

Keywords:Topic Modeling, Short text, Latent Dirichlet Allocation, Non-negative Matrix factorization, Semantic assisted NMF.

\section{Introduction}

Today's world has witnessed the global rise in the use of technology like Social Media tools .As a result of this lot of unstructured data is generated over the World Wide Web. This vast amount of electronic data can be made useful using text mining which is a process of extracting high-quality content from collections of documents. In order to acquire required information from this data, it is needed to identify the relevant documents. To mine such a pattern of words from a given set of documents, a statistical model called topic model is used [Likhitha et al. , (2019)]. Topic modeling is an unsupervised machine learning technique which helps in:

- Discovering hidden topical patterns that are present across the collection

- Annotating documents according to these topics

- These annotations are used in organizing, searching and summarizing text

To cluster words from a set of documents, topic modeling relies on the bag-of-words, their frequency and not on order of words[Alghamdi and Alfalqi, (2015)]. Various techniques used to obtain topic models are Latent Dirichlet Allocation, Non-negative Matrix Factorization, and Semantics-assisted Non-negative Matrix Factorization.

The rest of this paper is organized as follows; Section 2 presents various topic modeling methods. The experiments and results of topic model methods on ABCNews headline dataset are given in Section 3. Finally Section 4 concludes the paper. 


\section{Methods in Topic Modeling}

\subsection{Latent Dirichlet Allocation (LDA)}

LDA is probabilistic generative topic model used to identify topics in a given document. It works on the assumption that similar words are used to represent similar topics and each document as a mixture oftopics that represent whole corpus. The model considers that each word is mapped to at least one of the topic of document [Blei et al. ,(2003)].

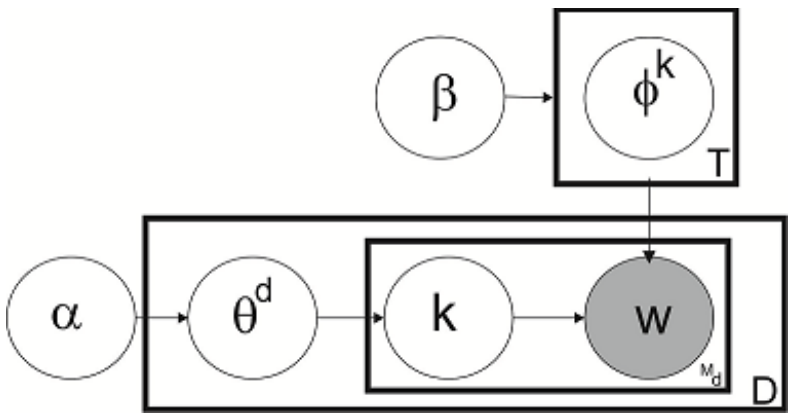

Fig. 1: Graphical Representation of LDA

In LDA documents are considered as probability distribution over topics and further each topic in a document as probability distribution over words. Having $\mathrm{T}$ topics, the probability distribution over $\mathrm{i}^{\text {th }}$ word( $(\mathrm{w})$ in a document is given as in Eq.(1),

$$
P\left(w_{i}\right)=\sum_{j=1}^{T} P\left(w_{i} \mid k_{i}=j\right) P\left(k_{i}=j\right)(1)
$$

where $k_{i}$ is the topic $j \in T$, which is a latent variable from which $i^{t h}$ word is taken. $P\left(w_{i} \mid k_{i}=j\right)$ is the probability of $i^{\text {th }}$ word; under topic $j . P\left(k_{i}=j\right)$ is the probability of choosing topic $j$ under given document, which can be different for various documents[Blei et al. ,(2003)];[Chhatbar,(2010)].

$P(w \mid k)$ can be represented as a set of $T$ multinomial distributions $\phi$ over $W$ unique words, such that $P(w \mid k=$ $j)=\phi_{w}^{j}$ and $P(k)$ as $D$ multinomial distribution $\theta$ over $T$ available topics, such that for each word in document $d \in D, \quad P(k=j \mid d)=\theta_{j}^{d}$. Multinomial distributions word-topic $(\phi)$ and topic-document $(\theta)$ are computed as [Chhatbar,(2010)]:

$$
\begin{gathered}
\theta_{j}^{(d)}=\frac{M_{d j}^{D T}+\alpha}{\sum_{t=1}^{T} M_{d t}^{D T}+T \alpha} \\
\phi_{i}^{(j)}=\frac{M_{i j}^{W T}+\beta}{\sum_{w=1}^{W} M_{w j}^{W T}+W \beta}
\end{gathered}
$$

Using Gibbs sampling, along with Dirichlet Prior on $\phi$ and $\theta$, probabilities can be estimated by following equation

$$
P\left(k_{i}=j \mid k_{-i,} W_{-i,} \ldots\right)=\frac{M_{d_{i} j}^{D T}+\alpha}{\sum_{t=1}^{T} M_{d_{i} t}^{D T}+T \alpha} \frac{M_{w_{i} j}^{W T}+\beta}{\sum_{w=1}^{W} M_{w j}^{W T}+W \beta}
$$

Here, considering probability of topic $k_{i}=j$ given all other word and document assignments. $\alpha$ is a prior weight of topic in a document, is initialized as $\alpha<1$ to prefer few topics per document. $\beta$ is a prior weight of word $w$ in topic, is initialized as very less than 1 to prefer fewer words per topic. $W_{-i}$ stands for all other word instances than the current one. $M^{W T}$ and $M^{D T}$ are matrices of counts for word-topic and document-topic assignments respectively. These steps are repeated till it reached a steady state where assignments are good. These assignments are then used to determine word-topic matrix which gives words from each document and document-topic matrix which yields belongingness of each document to particular topic [Chhatbar,(2010)].For a given collection of documents posterior distribution of the hidden variables determines topic-wise distribution of documents. These hidden variables are used in information retrieval and document browsing. LDA has achieved great results in modelling collection of normal length text like news article, research papers and blogs [Zuo et al., (2016)]. 


\subsection{Non-negative Matrix Factorization}

Non-negative matrix factorization is a linear algebraic model used for dimensionality reduction. This method is suitable where underlying factors are non-negative. As NMF yields good clustering results for high dimensional data, it is used for topic modeling [Yan et al.,(2013)];[ Choo et al.,(2013)]. Given N text documents, are represented as the term-document matrix in which each column represents a document and each element of matrix is the weight calculated through tf-idf [Kuang et al.,(2015)]. NMF decomposes given original matrix $D$ (term-document matrix) into two matrices $W$ (word-topic matrix) and $\mathrm{Z}$ (document-topic matrix) such that,

$$
D_{M \times N}=W_{M \times K} * Z_{K \times N}
$$

Consider a corpus with $N$ documents and $M$ distinct words in vocabulary. Term-document matrix is defined as $\mathrm{D} \in R_{+}{ }^{M \times N}$, where $R_{+}$denotes positive real numbers. Representation of bag of words of document $\mathrm{j}$ in terms of $M$ keywords is given by column vector $D_{(:, j)} \in R_{+}{ }^{M \times I}$. The term-document matrix is $D \approx W Z^{T}$, where $W \in$ $R_{+}{ }^{M \times K}$ and $Z \in R_{+}{ }^{N \times K}, K<<\min (M, N)$ is the number of latent factors (i.e., topics). Usually, this approximation can be devised as in Eq. (6) [Choo et al.,(2015)]:

$$
\min _{W, Z \geq 0}\left\|D-W Z^{T}\right\|_{F}^{2}
$$

Elements in column vector of term-topic matrix $W_{(:, k)} \in R_{+}{ }^{M x l}$ are weights of $M^{\text {th }}$ keyword under $K^{\text {th }}$ topic , and row vector $W_{(i,)} \in R_{+}{ }^{l \times K}$ is semantic representation of word $i$. Row vector of document-topic matrix $Z_{(j,:)} \in$ $R_{+}{ }^{1 \times K}$ represents weights for $j^{\text {th }}$ document corresponding to $K^{\text {th }}$ topic. As short text is very sparse, the factor matrices $W$ and $Z$ are updated using Block Coordinate Descent (BCD) algorithm. It is a divide-and-conquer strategy which divides data in blocks and updates data block by block [Kim et al.,(2014)].

Update W:

$$
W_{(:, k)}=\frac{W_{(:, k)}+(D Z)_{(:, k)}-\left(W Z^{T} Z\right)_{(:, k)}}{\left(Z^{T} Z\right)_{(k, k)}}
$$

Update Z:

$$
Z_{(:, k)}=\frac{Z_{(:, k)}+\left(D^{T} W\right)_{(:, k)}-\left(Z W^{T} W\right)_{(:, k)}}{\left(W^{T} W\right)_{(k, k)}}
$$

The association between different documents strongly depends on keywords and vice-versa. In short text each document has few keywords and NMF does not consider semantic relationship between keywords, hence clustering performance is poor [Shi et al.,(2018)].

\subsection{SeaNMF Model}

The Semantic-assisted NMF (SeaNMF) is a method which reveals semantic relationship between keywords and their context to discover topics from short text. During training, word embedding is used to find semantic association between keyword and their contexts. Semantic correlation matrix(S) between word-context is obtained from vocabulary of words $(V)$ using skip gram model [Levy and Glodberg,(2014)];[ Milolov et al.,(2013)].

$$
S_{i j}=\left[\log \left(\frac{\#\left(w_{i}, c_{j}\right)}{\#\left(w_{i}\right) \cdot p\left(c_{j}\right)}\right)\right]
$$

where $\mathrm{w}_{\mathrm{i}} \in V, c_{j} \in V,, \mathrm{p}\left(\mathrm{c}_{\mathrm{j}}\right)$ is a distribution of words based on its frequency in corpus.

In SeaNMF algorithm bag-of-word representation is used to construct term-document matrix $(D)$. Then, semantic correlation matrix $S$ is computed by Eq.(9). The latent factor matrices of words $(W)$, Context $\left(W_{c}\right)$ and documents $(Z)$ are randomly initialized with non-negative numbers. In each iteration, their weights are updated by using Block Coordinate Decent (BCD) algorithm [Kim et al.,(2014)]. Updated weights $W_{(:, k)}$ and $W_{c(:, k)}$ are normalized by $\mathrm{F}$-norm. Process is repeated until the algorithm converges [Shi et al.,(2018)]. The $W, W_{c}, Z$ are updated for k topics as in Eq. (10) and (11) :

Update $\mathrm{W}$

$$
W_{(:, k)}=\frac{W_{(:, k)}+(D Z)_{(:, k)}+\alpha\left(S W_{c}\right)_{(;, k)}-\left(W Z^{T} Z\right)_{(:, k)}-\alpha\left(W W_{c}^{T} W_{c}\right)_{(:, k)}}{\left(Z^{T} Z\right)_{(k, k)}+\alpha\left(W_{c}^{T} W_{c}\right)_{(k, k)}}
$$

Update Wc

$$
W_{c(:, k)}=\left[W_{c(:, k)}+\frac{\left.(s W)_{(:, k)}-\left(W_{c} W^{T} W\right)_{(:, k)}\right)}{\left(W^{T} W\right)_{(k, k)}}\right]
$$


where $\alpha \in R_{+}$is ascale parameter.

Consideration of word-context semantic relationship in the overall updating procedure yields highly correlated top keywords under each topic.

\section{Results and Discussion}

\subsection{Dataset Used}

Experimentation is carried out on the ABC Millions Headlines dataset published on Kaggle, sourced from Australian Broadcasting Corporation (ABC). This contains Million news headlines with published date and headline text. It includes the entire corpus of articles published by the $\mathrm{ABC}$ website in the given time range 2003-2019 with focus on international news. Headlines from various domains like financial crisis, Iraq war, various elections, ecological disasters, terrorism, famous people, local crimes etc. are covered [Kulkarni (2017)].

\subsection{Results}

This section shows performance of various topic modeling algorithms on ABCNews dataset.

For experimentation 2,57,848 headlines are considered. Dataset is preprocessed which includes tokenization, removal of stop words, lemmatization [Vijayarani et al.,(2016)]. This creates dictionary of words. It's tf-idf score is calculated and given as input to LDA, NMF and SeaNMF model. Topics discovered by these topic modeling methods are listed in Table 1, Table 2 and Table 3.

\begin{tabular}{|l|l|l|l|l|l|l|l|l|l|}
\hline Topic_1 & Topic_2 & Topic_3 & Topic_4 & Topic_5 & Topic_6 & Topic_7 & Topic_8 & Topic_9 & Topic_10 \\
\hline Cup & South & search & High & killed & claims & set & govt & Water & police \\
\hline world & Talks & continues & Rise & crash & inquiry & opposition & council & Probe & man \\
\hline decision & Howard & missing & market & two & rejects & gold & plan & Group & court \\
\hline Win & Qld & family & Anti & day & New & return & urged & Ban & face \\
\hline takes & Iraq & open & Year & dead & Aid & coast & health & Safety & charged \\
\hline Toll & North & Body & Price & three & Govt & attacks & boost & New & murder \\
\hline Test & Aust & accused & workers & one & Chief & clash & funds & Call & drug \\
\hline Rate & West & sought & Strike & attack & drought & abuse & new & Council & death \\
\hline Park & East & Lead & Prices & injured & Fire & iraqi & indigenous & Warning & charges \\
\hline australia & Blaze & First & expected & hopes & farmers & debate & public & Prompts & Case \\
\hline
\end{tabular}

Table 1: Top 10 keywords for 10 topics discovered by LDA

Table 2: Top 10 keywords for 10 topics discovered by NMF

\begin{tabular}{|l|l|l|l|l|l|l|l|l|l|}
\hline Topic_1 & Topic_2 & Topic_3 & Topic_4 & Topic_5 & Topic_6 & Topic_7 & Topic_8 & Topic_9 & Topic_10 \\
\hline iraq & war & police & Govt & man & new & says & council & Iraqi & world \\
\hline troops & anti & death & Nsw & court & plan & qld & water & Baghdad & cup \\
\hline baghdad & plan & anti & Qld & death & water & troops & plan & Troops & win \\
\hline war & world & nsw & Plan & qld & court & baghdad & claims & Claims & claims \\
\hline claims & Nsw & claims & claims & water & qld & win & qld & Plan & rain \\
\hline win & Win & water & water & win & nsw & rain & rain & Rain & death \\
\hline world & claims & win & death & rain & baghdad & death & court & Water & water \\
\hline water & iraqi & qld & Rain & nsw & death & court & win & Death & anti \\
\hline death & govt & rain & Anti & world & win & water & world & War & nsw \\
\hline man & baghdad & troops & court & govt & claims & cup & iraq & World & police \\
\hline
\end{tabular}


Table 3: Top 10 keywords for 10 topics discovered by SeaNMF

\begin{tabular}{|l|l|l|l|l|l|l|l|l|l|}
\hline Topic_1 & Topic_2 & Topic_3 & Topic_4 & Topic_5 & Topic_6 & Topic_7 & Topic_8 & Topic_9 & Topic_10 \\
\hline new & Govt & police & Council & downer & Man & win & Weather & market & killed \\
\hline us & urged & probe & Plan & un & Court & edge & Damage & Prices & kills \\
\hline council & Plan & investigate & Govts & blair & charged & draw & Cyclone & Stocks & least \\
\hline laws & Nsw & man & Proposal & annan & murder & finals & Ses & Profit & dead \\
\hline plan & Vic & missing & Proposed & peace & Jailed & blues & firefighters & markets & blast \\
\hline zealand & Says & crash & Mp & pm & charges & tigers & Winds & Asx & kill \\
\hline centre & Wa & search & Consultation & powell & Guilty & final & Flooding & higher & baghdad \\
\hline chief & Qld & car & Councilor & eu & Bail & match & Storms & profits & bomb \\
\hline iraq & funds & seek & Unhappy & bush & alleged & clash & Flood & Price & kashmir \\
\hline may & Fire & death & Education & iran & Teen & play & Crews & Sales & wounded \\
\hline
\end{tabular}

\subsection{Topic Model Evaluation}

Evaluation of topic model is done by topic coherence [Ramirez and Brena(2011)]; Cilibrasi and Vitanyi (2001)]. There are two measures in topic coherence Intrinsic and Extrinsic. Intrinsic measure needs ordered word set as it compares word with its preceding and succeeding words. In extrinsic measure every word is paired with every other word under given set [Alhawarat and Hegazi (2018)];[Stevens and Kegelmeyer(2012)];[Roder and Both(2015)].

The Normalized Google Distance (NGD) is an extrinsic semantic similarity measure derived from the number of hits returned by the Google search engine for a given set of keywords[ Cohen and Vitanyi(2013)]. Keywords with similar or same meaning in natural language sense tend to be "close" in units of NGD, while words with dissimilar meaning tend to be farther apart. The NGD score is calculated by the formula:

$$
N G D(x, y)=\frac{\max \{\log f(x), \log f(y)\}-\log (x, y)}{\log M-\min \{\log f(\mathrm{x}), \log f(y)\}}
$$

where $f(x)$ and $f(y)$ are frequency of term $x$ and term $y, M$ is overall number of web pages indexed by Google. NGD(x,y) is a nonnegative score [Alguliev et al. ,(2011)].

The set of Keywords under each topic obtained after execution of LDA, NMF and SeaNMF are compared using NGD. Following results are obtained:
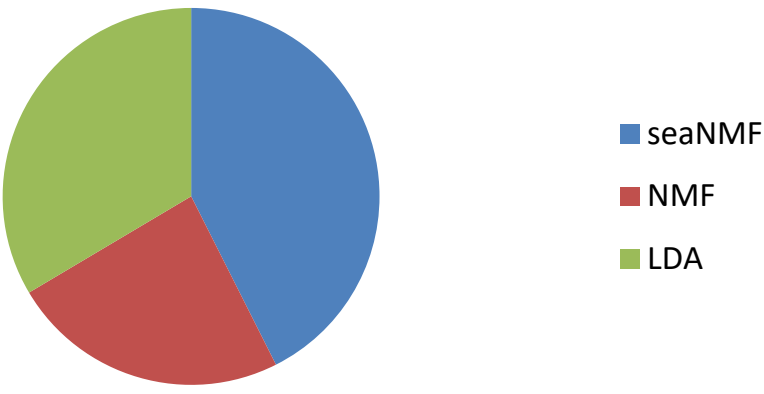

Fig 2: Number of correlated terms

The terms having NGD greater than 0 and less than 1 are said to be correlated terms.[Cilibrasi and Vitanyi (2001)] Fig. 2 shows SeaNMF is giving more correlated terms as compare to NMF and LDA.

Degree of correlativity of keywords is calculated by computing averaged NGD score per topic for all mentioned methods, results are listed in Table 4: 
Table 4: Average NGD of 10 Topics

\begin{tabular}{|c|c|c|c|}
\hline Topic & SeaNMF & NMF & LDA \\
\hline 1 & 0.8905 & 0.6897 & 0.5529 \\
\hline 2 & 0.8332 & 0.6397 & 0.3504 \\
\hline 3 & 0.6167 & 0.4242 & 0.5014 \\
\hline 4 & 0.5226 & 0.7585 & 0.5163 \\
\hline 5 & 0.8643 & 0.5032 & 0.6776 \\
\hline 6 & 0.6194 & 0.5933 & 0.8502 \\
\hline 7 & 0.7359 & 0.4223 & 0.7262 \\
\hline 8 & 0.6577 & 0.6186 & 0.7947 \\
\hline 9 & 0.5372 & 0.7205 & 0.3189 \\
\hline 10 & 0.5105 & 0.4663 & 0.6434 \\
\hline Average & $\mathbf{0 . 6 7 8 8}$ & $\mathbf{0 . 5 8 3 6 3}$ & $\mathbf{0 . 5 9 3 2}$ \\
\hline
\end{tabular}

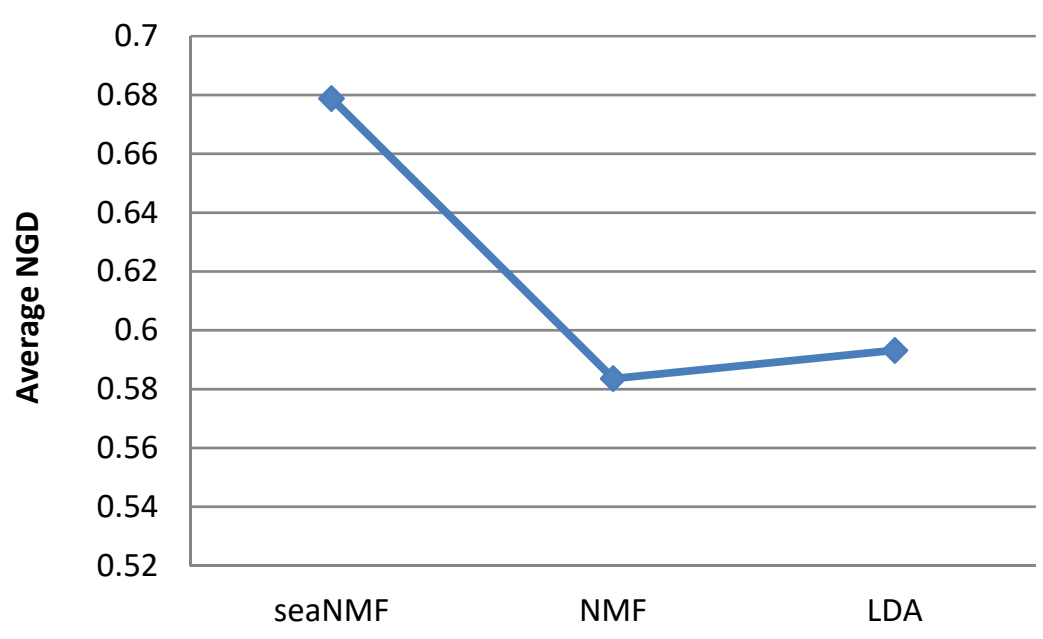

Fig 3: Average NGD for 10 Topics

The degree of correlativity of keywords computed is $67.88 \%, 58.6 \%, 59.32 \%$ for SeaNMF, NMF and LDA respectively. Fig. 3 shows that words clustered under each topic by SeaNMF are highly correlated.

\section{Conclusions}

Learning meaningful topics from short text is considered to be a challenge due to limited contextual information in it. This paper includes empirical study of three state-of- the-art methods of topic modeling. LDA is good for normal length text but not so for short text as it does not consider the relationships among keywords during topic discovery. NMF is a dimension reduction technique which yields clustering results based on the words in same region using term-document matrix whereas SeaNMF gives grouping of words using word-context semantic correlation matrix and skip-gram view of corpus that reveals word semantic association.SeaNMF outperforms NMF and LDA as it discovers more relevant topics from short text. 


\section{References}

[1] Alghamdi Rubayyi, Khalid Alfalqi, (2015), "A Survey of Topic Modeling in Text Mining”, International Journal of Advanced Computer Science and Applications, vol. 6, no. 1, pp 147-153

[2] Alguliev Rasim, Aliguliyev Ramiz, Makrufa S Hajirahimova, Chingiz A Mehdiyev,(2011) "MCMR: Maximum Coverage and Minimum redundant text summarization model", Article in Expert Systems with Applications, Elsevier.

[3] Alhawarat M., Hegazi M.(2018), "Revisiting K-means and topic modeling, a comparison study to Cluster Arabic Documents", IEEE Access.

[4] Blei D, A. Ng, M. Jordan,(2003),“Latent Dirichlet Allocation”, Journal of Machine Learning Research, 3: 993-1022

[5] Chhatbar Cirag Dilip, (2010), "Improving Statistical Topic Models by Using Ontological Concepts", COMP8740 Project Report, Dept., Computer Science, Australian National University.

[6] Choo Jaegul, Changhyun Lee, Chandan K. Reddy, Haesun Park,(2013), "Utopian:User-driven Topic Modeling based on Interactive Non-negative Matrix Factorization", IEEE transactions on Visualization and Computer Graphics, vol.19

[7] Choo Jaegul, Changhyun Lee, Chandan K. Reddy, Haesun Park, (2015) "Weakly supervised nonnegative matrix factorization for userdriven clustering", Data Mining and Knowledge Discovery, 1598-1621.

[8] Cilibrasi Rudi, Vitanyi Paul,(2001) “Automatic Meaning Discovery Using Google”, BSIK/BRICKS project, Netherland

[9] Cohen Andrew R. and Paul M. B. Vitanyi,(2013), "Normalized Google Distance of Multisets with Applications", CoRR, abs $\mid 1308.3177$

[10] Kim Jingu, Yunlong He,and Haesun park,(2014), “Algorithms for nonnegative matrix and tensor factorizations: A unified view based on block coordinate descent framework", Journal of Global Optimization 58, 2(2014), 285-319

[11] Kuang Da, Jaegul Choo, and Haesun Park,(2015) "Nonnegative matrix factorization for interactive topic modeling and document clustering", In Partitional Clustering Algorithms, Springer, 215 - B

[12] Kulkarni Rohit,(2017), A Million News Headlines [CSV Data file], doi:10.7910/DVN/SYBGZL, Retrieved from: https://www.kaggle.com/therohk/million-headlines

[13] Levy Omer and Goldberg Yoav,(2014), "Neural Word Embedding as Implicit Matrix Factorization". In Advances in Neural Information Processing Systems 27. Curran Associates, Inc., 2177-2185.

[14] Likhitha S. , B. S. Harish, H. M. Keerthi Kumar,(2019), "A Detailed Survey on Topic Modeling for Document and Short Text Data", International Journal of Computer Applications, vol. 178, no. 39

[15] Milolov Tomas, Chen Kai, Corrado Greg, Dean Jeffrey,(2013), "Efficient Estimation of word representation in vector space", arXiv preprint arXiv: 1301.3781

[16] Ramirez Eduardo H., Brena Ramon,(2011), “Topic Model Validation”, Elsevier.

[17] Roder Michael, Both Andreas, (2015),s "Exploring the space of topic coherence Measures", ACM

[18] Shi Tian, Kyeongpil Kang, Jaegul Choo, Chandan Reddy, (2018),"Short-Text Topic Modeling via Non-negative Matrix Factorization Enriched with Local Word-Context Correlations", ACM ISBN 978-1-4503-5639-8.

[19] Stevens Keith, Kegelmeyer Philip, (2012), "Exploring Topic Coherence over many models and many topics", proceeding of 2012 joint conference on Empirical Methods in Natural Language Processing and Computational Natural Language Learning, pages 952-961

[20] Dr. Vijayarani S., J. Ilamathi, Ms. Nithya , (2016), “ Preprocessing Techniques for Text Mining-An Overview”, International Journal of Computer Science \& Communication Networks, vol 5.

[21] Yan Xiaohui, Jiafeng Guo, Shenghua Liu, Xueqi Cheng, Yanfeng Wang,(2013), "Learning Topics in short Text by Non-negative Matrix Factorization on Term Correlation Matrix", In Proceedings of the SIAM international Conference on Data Mining.

[22] Zuo Yuan, Junjie Wu, Hui Zhang, Hao Lin, Fei Wang, Ke Xu, Hui Xiong, (2016),"Topic Modeling for Short Text: A PseudoDocument View", KDD'16, ACM, August 13-17 